\title{
AspectLISA: an aspect-oriented compiler construction system based on attribute grammars
}

\author{
Damijan Rebernak, Marjan Mernik 1,2 \\ University of Maribor \\ Faculty of Electrical Engineering and Computer Science \\ Smetanova ul. 17, 2000 Maribor, Slovenia \\ Pedro Rangel Henriques ${ }^{3}$ \\ University of Minho \\ Department of Computer Science \\ Campus de Gualtar \\ 4710 - 057 Braga, Portugal \\ Maria João Varanda Pereira ${ }^{4}$ \\ Polytechnic Institute of Bragança \\ Campus de Sta. Apolónia \\ Apartado 134 - 5301-857, Bragança, Portugal
}

\begin{abstract}
The use of object-oriented techniques and concepts, like encapsulation and inheritance, greatly improves language specifications towards better modularity, reusability and extensibility. Additional improvements can be achieved with aspect-oriented techniques since semantic aspects also crosscut many language constructs. Indeed, aspect-oriented constructs have been already added to some language specifications.
\end{abstract}

The LISA compiler construction system follows an object-oriented approach and has already implemented mechanisms for inheritance, modularity and extensibility. Adding aspects to LISA will lead to more reusable language specifications. In the paper, aspectoriented attribute grammars are introduced, and the underlying ideas are incorporated into AspectLISA, an aspect-oriented compiler generator based on attribute grammars.

Key words: Attribute grammars, aspect-oriented programming, compiler generators. 


\section{Introduction}

The challenge in programming language definition is to support modularity and abstraction in a manner that supports reusability and extensibility. A language designer often wants to include new language features incrementally as the programming language evolves. This is especially true in developing domain-specific languages (DSLs) which change more frequently than general-purpose programming languages [17]. Ideally, a language designer would like to build a language simply by reusing different language definition modules (e.g., language components), such as modules for expressions, declarations, etc., regardless of the different formal methods that may be used to specify such language components. This approach is common in component-based programming [24] where components can be simply plug-ins.

This cannot be done now, even if we restrict ourselves to just one of the formal methods (abstract state machines, action semantics, algebraic specifications, attribute grammars, denotational semantics, operational semantics, two-level grammars, etc. [23]) since different compiler-compilers (automatic compiler generation systems) use different and incompatible specification languages (e.g., despite the fact that Eli [6] and FNC-2 [9] both rely on attribute grammars one can not exchange language definition modules written in the other system). Moreover, the same is usually true even in the case of the same specification language since syntax entities (e.g., non-terminals and terminals) and semantic entities (e.g., attributes and semantic rules in the case of attribute grammars) are not constituents of the hidden part of the module, nor are the parameters of language definition modules. For example, when importing a module for expressions some non-terminals may clash with existing non-hidden non-terminals producing undesirable effects. Such a module can be parameterized using non-terminals as parameters to solve renaming problems. However, modules with dozens of parameters are hard to use.

Compared to modern programming languages, such as object-oriented or functional languages, language specifications of the 1980's and early 1990's were far less advanced, specifically concerning provisions for abstraction, modularization, extensibility and reusability. Recently, concepts from general programming languages have been successfully incorporated into language specifications. Among them, object-oriented techniques are one of the most successful. Indeed, this had several benefits on language specifications. To fully achieve modularity, extensibility and reusability these techniques need to be combined with aspect-oriented techniques because semantic aspects also crosscut many language constructs [19]. These observations have been taken into account in extending the LISA specification language [18] with aspect-oriented features. The paper presents AspectLISA,

\footnotetext{
1 This work is sponsored by bilateral project BI-PT/04-06-008 "Grammar Based Systems" between Slovenia and Portugal.

2 Email: \{damijan.rebernak, marjan.mernik\}@uni-mb.si

3 Email: prhedi.uminho.pt

4 Email: mjoao@ipb.pt
} 
which is an aspect-oriented compiler generator based on attribute grammars.

The paper is organized as follows. A brief introduction to aspect-oriented programming is given in section 2 followed by related work presented in section 3 . The main part of the paper constitutes section 4 where AspectLISA is discussed. A small case study illustrating ideas is given in section 5 . The concluding comments are mentioned in section 6 .

\section{Aspect-oriented programming}

The major abstraction technique in software engineering is to divide the system into functional components in such manner that changes to a particular component do not propagate through the entire system [5,22]. However, some issues, called aspects, are system wide and cannot be put into a single functional component. As examples, failure handling, persistence, communication, coordination, memory management, are aspects of a system behavior that tend to crosscut groups of functional components. As a consequence, functional components are tangled with aspect code. This tangling problem makes functional components less reusable and difficult to develop, understand, and evolve. A solution is provided by aspectoriented programming (AOP) [12] which is a programming technique for modularizing concerns that crosscut the basic functionality of programs. In AOP, aspect languages are used to describe properties which crosscut basic functionality in a clean and a modular way. Despite that the main part of AOP research is devoted to general-purpose languages $[11,16]$ similar problems exists in domain-specific languages. For example, in language specifications modularization is usually based on language syntax constructs, whereas the modularization based on different aspects (e.g. name analysis, type checking, code generation, etc.) would be more beneficial. To overcome this problem aspect-oriented techniques can be used.

In order to achieve the desired properties of the system, we need an aspect weaver that combines the component and the aspect language by weaving advice at appropriate join points and may involve merging components, modifying and optimizing them.

\section{Aspects in language development}

Aspect-oriented programming is a very promising approach and has been successfully used in tools for language definition and implementation $[4,7,10,13,15,26,27]$. In this context, aspects have been used for many different tasks (e.g., in [26] an extension for weaving debugging information into DSL specifications is reported). In the rest of this section we describe in more detail some of the more relevant contributions in the field, using aspects in language specification or implementation. 


\subsection{JastAdd}

JastAdd [7] is a Java-based system for compiler construction. JastAdd is centered around object-oriented representation of the abstract syntax tree (AST). Nonterminals act as abstract super classes and productions act as specialized concrete subclasses that specify the syntactic structure, attributes and semantic rules. All these elements can be inherited, specialized, and overridden in subclasses. The idea of aspect-orientation in JastAdd is to define each aspect of the language in a separate class and then weave them together at appropriate places. The JastAdd system is a class weaver: it reads all the JastAdd modules and weaves the fields and methods into the appropriate classes during the generation of the AST classes. With separation of different language aspects among different classes, developers have the possibility to use all features of Java to specify aspects. In the following example, taken from [7], two different aspects are described in separate classes. The first one (typechecker. jadd) performs type checking for expressions and computes the boolean field typeError. The unparser. jadd (second example) implements an unparser which makes use of the field typeError to report type-checking errors.

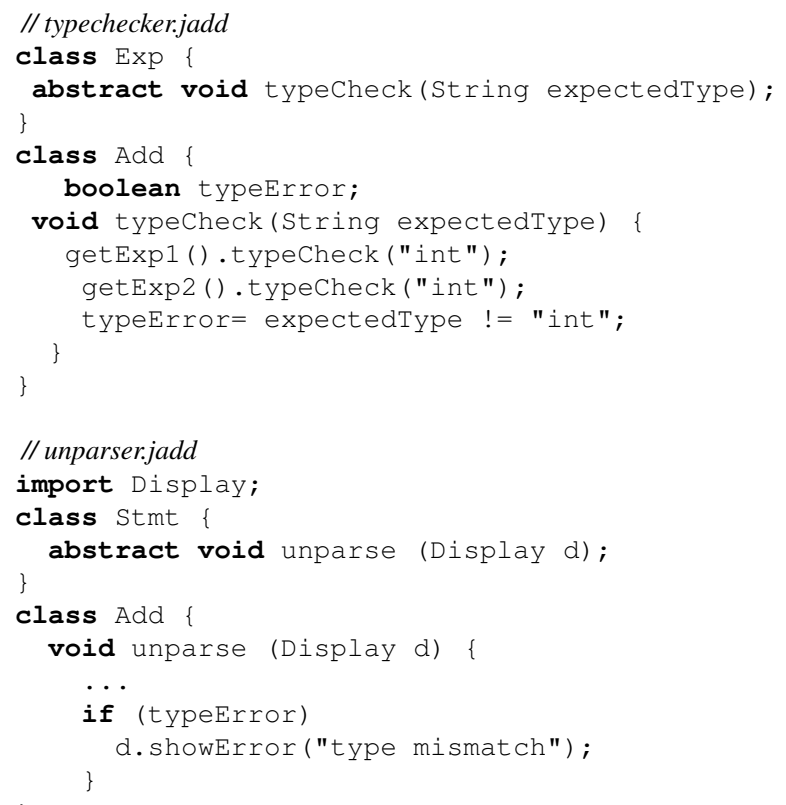

Every field, method, import declaration is weaved to all generated AST classes, as can be seen in following example.

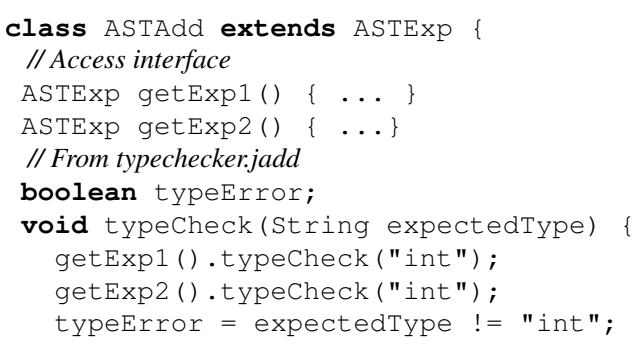




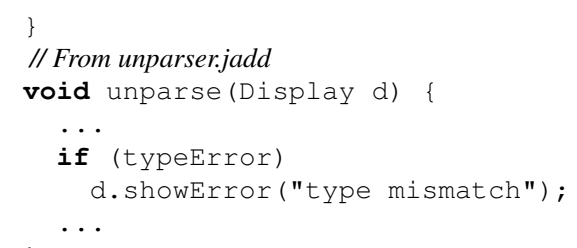

As can be seen in the example above, this approach does not follow the conventional AOP join point model (JPM) where join points are specified using a pointcut pattern language. However, it can be seen as inter-type declarations in AspectJ [11] where join points are all non-anonymous types in the program and pointcuts are the names of classes or interfaces.

\subsection{Aspect $G$}

To generate an additional language-based tool (e.g., debugger) new specifications need to be added in several places in language specifications [8]. These new additions can be seen as aspects (e.g., debugging aspects). It was observed [26] that such aspects crosscut basic language specifications. Hence, the aspect-oriented language AspectG [2] was created for modular implementation of crosscutting concerns in the ANTLR language definition [1]. Since ANTLR belongs to syntax directed translations (semantic rules are not declaratively specified and order of semantic rules is important) AspectG uses the following model:

- join points are static points in language specifications where additional aspects can be weaved,

- pointcuts specify join points and include not only the syntax level of the grammar but also the semantics associated with a particular syntax (see within and match constructs in the example below),

- advice are similar to AspectJ notion (before and after) and brings together a pointcut and a body of code.

An example of pointcut and advice in AspectG is shown below.

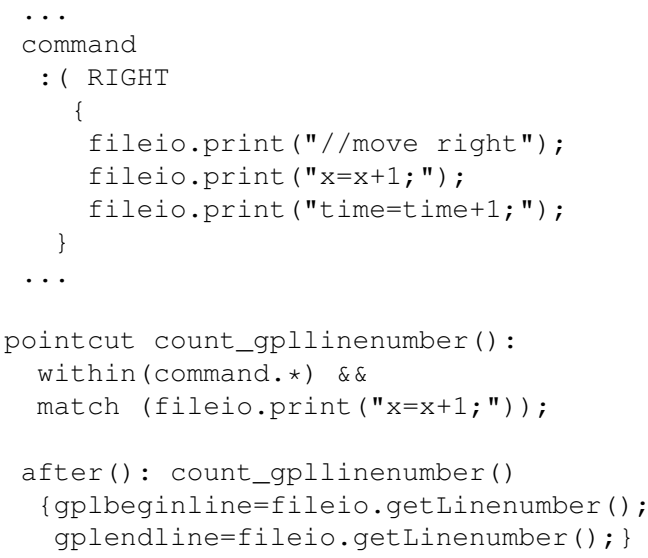


The result of weaving is:

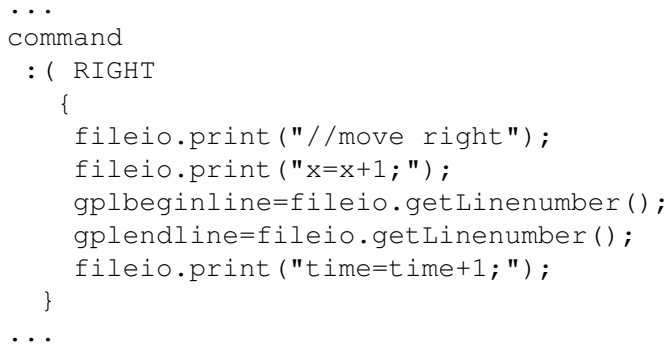

\subsection{AspectASF}

AspectASF [13] is a simple aspect language for language specifications written in the ASF+SDF [25] formalism. Only rewrite rules are supported. Therefore, join points in AspectASF are static points in equation rules describing semantics of the language. The pointcut pattern language in AspectASF is a very simple pattern matching language on the structure of equations where only labels and left-hand sides of equations can be matched. Pointcuts can be of two types: entering an equation (after a succesfull match of left-hand side) and exiting an equation (just before returning the right-hand side). Examples (all examples in this section are taken from [13]) of pointcuts in AspectASF language are:

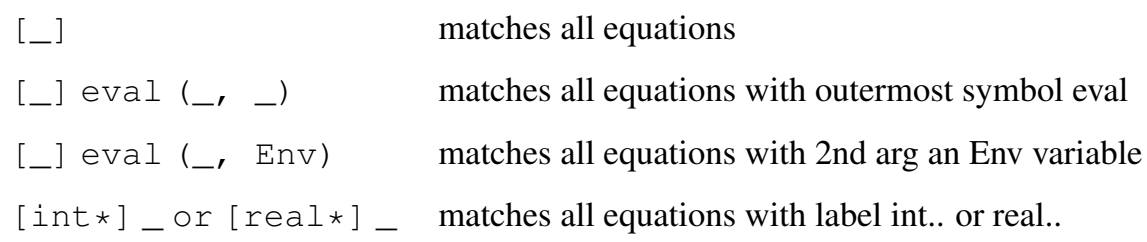

Advice code specify additional equations which are written in the ASF formalism. There are two types of advice: after entering an equation (concatenating equations to the beginning of the list of equations that is matched by the pointcut) and before exiting an equation (concatenating equations to the end of the list of equations that is matched by the pointcut). An example of AspectASF is shown below.

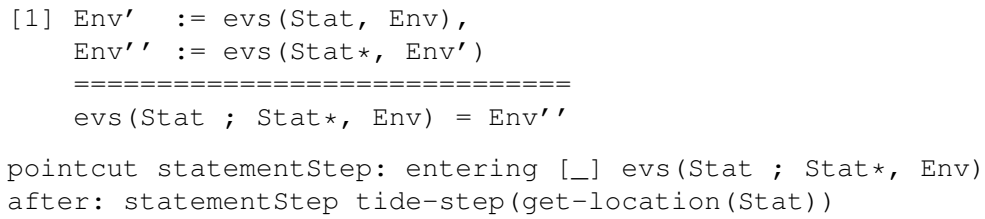

After weaving takes place the aspects are weaved into the original language specifications. In other words, additional equations are appended to appropriate places.

[1] tide-step (get-location (Stat)),

Env' := evs (Stat, Env)

Env' ${ }^{\prime}:=\operatorname{evs}($ Stat*, Env')

evs (Stat; Stat*, Env) = Env' ' 


\section{AspectLISA}

\subsection{Introduction to LISA}

In the LISA project $[18,20]$, one of the main goals was to enable incremental language development. It was soon recognized that inheritance can be very helpful since it is a language mechanism that allows new definitions to be based on the existing ones. A new specification can inherit the properties of its ancestors, and may introduce new properties that extend, modify or defeat its inherited properties. In object-oriented languages the properties that consist of instance variables and methods are subject to modification. The corresponding properties in language definitions based on attribute grammars are:

- lexical regular definitions,

- attribute definitions,

- rules which are generalized syntax rules that encapsulate semantic rules, and

- operations on semantic domains.

Therefore, regular definitions, production rules, attributes, semantic rules and operations on semantic domains can be inherited, specialized or overridden from ancestor specifications. In this approach the attribute grammar as a whole is subject to inheritance employing the "Attribute grammar = Class" paradigm [21].

A very simple language for moving a robot can illustrate our incremental language development approach [20]. The language for robot movement is defined in Fig. 1. The robot can move in different directions and the task is to compute its final position. Over time, the language is extended with new features. For example, we would like to know when the robot will reach the final position. The new language (RobotTime) is specified as an extension of the Robot language (Fig. 2). This is a good example of how different aspects can be modularized in our approach. In the Robot language just the semantic rules for robot movement have been described, while the RobotTime language contains just the semantic rules for time calculation. The RobotTime language inherits regular definitions, syntax constructs and semantic rules from the Robot language and adds new semantic rules for time calculation. Note that the same effect is obtained by implicit pointcuts in aspect-oriented systems like JastAdd [7] (see section 3).

As already mentioned, object-oriented techniques and concepts need to be combined with aspect-oriented techniques to achieve better modularity, extensibility and reusability. This issue is further described in the following sections.

\subsection{Aspect-oriented Attribute Grammars}

Aspect-oriented attribute grammar (AspectAG) is an attribute grammar [14] extended with pointcut and advice specifications [12], Aspect $A G=(G, A, R, P c$, $A d)$. Context-free grammar $G=(N, T, S, P)$, set of attributes $A$, and set of semantic rules $R$ have the same standard meaning of attribute grammars, as for example described in [18]. 


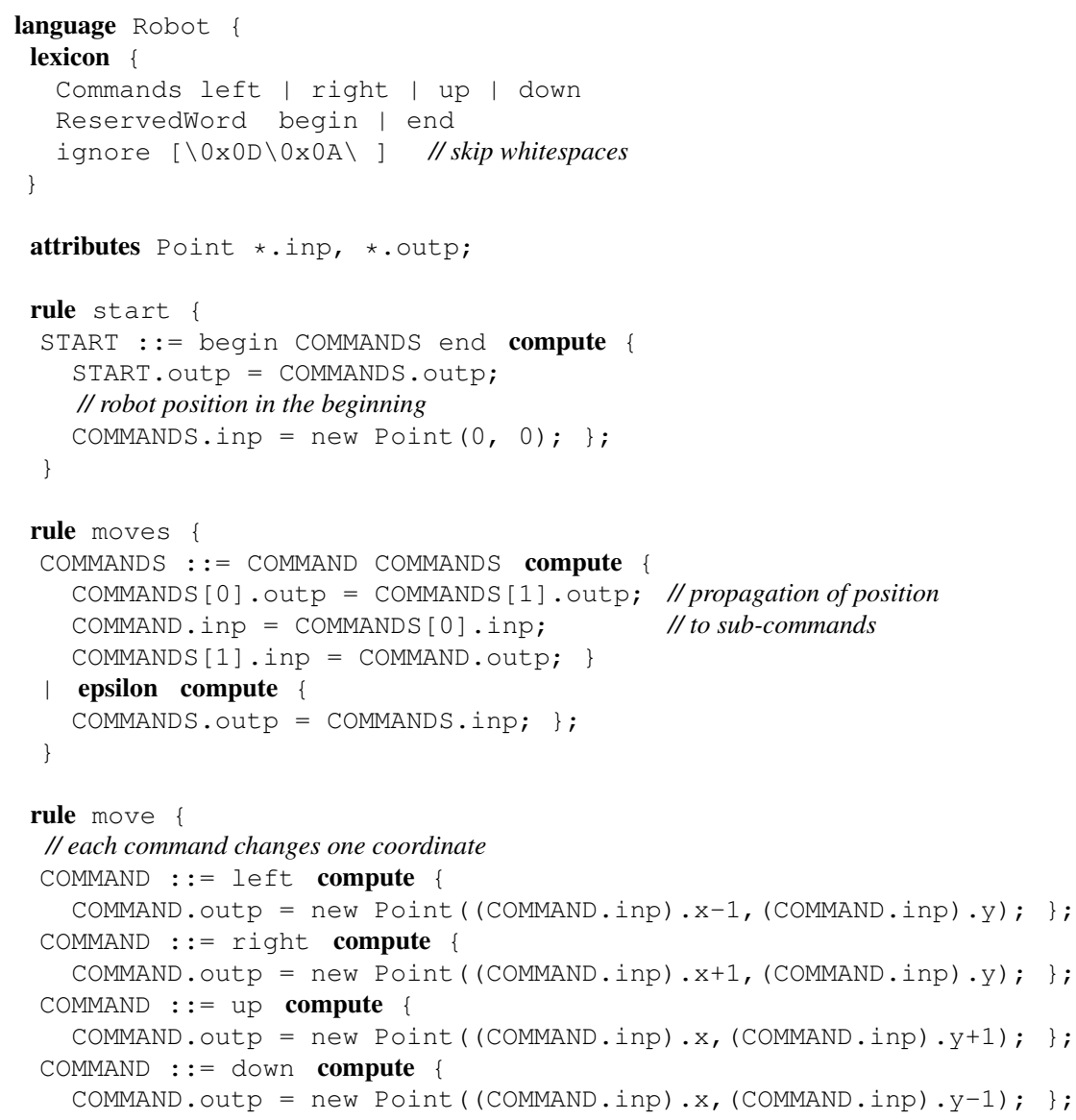

Fig. 1. Robot Language using LISA

Pointcuts $P c$ is a set of pointcut productions, $P c=\left\{p c_{1}, \ldots, p c_{m}\right\}$, where pointcut production $p c_{i}, 1 \leq i \leq m$, has the following form:

$$
p c_{i}<X_{1}, \ldots, X_{r}>: L H S \rightarrow R H S
$$

In pointcut production $p c_{i}$ special wildcard symbols $(. ., *)$ can be used. Wildcard symbol '*' denotes a symbol or some part of its name and can be used in the $L H S$ and $R H S$. Wildcard symbol '..' denotes zero or more symbols and can be used only in the $R H S$. Symbols $X_{i}, 1 \leq i \leq r$, are symbols from $L H S$ and $R H S$ and denote the public interface for advice. A pointcut production $p c_{i}<X_{1}, \ldots, X_{r}>$ : $L H S \rightarrow R H S$, selects a production $p: X_{0} \rightarrow X_{1} \ldots X_{n} \in P$ if $X_{0}$ matches $L H S$ and $X_{1} \ldots X_{n}$ match $R H S$. Let $P m_{i}$ denote the set of productions selected by pointcut production $p c_{i}, P m_{i}=\left\{p_{i} \mid p_{i} \in P\right.$ and $p_{i}$ is matched by $\left.p c_{i}\right\}$. Matched productions $P m$ selected by pointcuts $P c$ is then defined as $P m=\bigcup_{i=1 . . m} P m_{i}$, $P m \subseteq P$. To match productions $P m$, additional semantic rules specified in advice $A d$ are attached.

$A d$ is a set of advice, $A d=\left\{a d_{1}, \ldots, a d_{l}\right\}$, where advice $a d_{k}, 1 \leq k \leq l$, has 


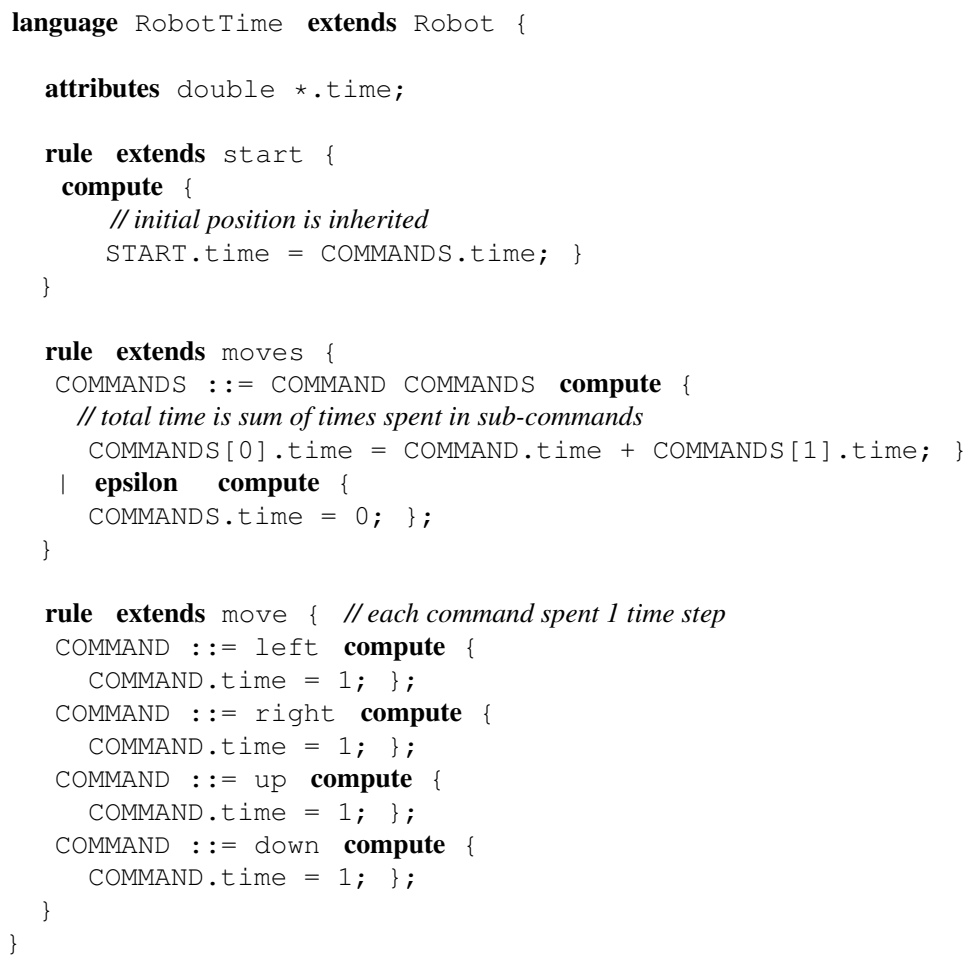

Fig. 2. RobotTime Language using LISA

the following form:

$$
a d_{k}<S_{1}, \ldots, S_{r}>\text { on } p c_{i}\left\{R s_{k}\right\}
$$

Semantic rules $R s_{k}$ has the following form:

$$
R s_{k}=\left\{S_{j} . a=f\left(y_{1}, \ldots, y_{k}\right) \mid a \in A\left(S_{j}\right), y_{i} \in\left(A\left(S_{1}\right) \cup \ldots \cup A\left(S_{r}\right)\right), 1 \leq i \leq k\right\}
$$

Defining attributes attached to symbols $S_{j}, 1 \leq j \leq r$, are defined by semantic rules in $R s_{k}$. Advice $a d_{k}$ is applied on pointcut $p c_{i}$, which match productions $P m_{i}$. For each match production $p_{i} \in P m_{i}$, the actual set of semantic rules $R a_{k i}$ is obtained by replacing formal symbols $S_{j}$ (specified in $a d_{k}$ ) by actual symbols $X_{j}$ (specified in $p c_{i}$ ) in $R s_{k}$. The set of semantic rules $R a$ obtained from advice $A d$ and pointcuts $P c$ is defined as $R a=\bigcup_{k=1 . ., i=1 . . m} R a_{k i}$ and needs to be merged with ordinary semantic rules $R p_{i}$, to obtain well defined attribute grammar $A G=\left(G, A, R^{\prime}\right)$ in the following manner: $R p_{i}^{\prime}=R p_{i} \cup\left(\bigcup_{k=1 . . l} R a_{k i}\right), R^{\prime}=\bigcup_{i=1 . . n} R p_{i}^{\prime}$. Note that $(G, A, R, P c, A d)=\left(G, A, R^{\prime}\right)$. Therefore, an aspect-oriented attribute grammar is an attribute grammar where some semantic rules are not attached explicitly to production rules but implicitly as advice into productions selected by pointcuts. When semantic rules are merged, only one semantic rule for each defining attribute must exist, otherwise the attribute grammar is not well defined [14]. The following 
illustrates a simple example:

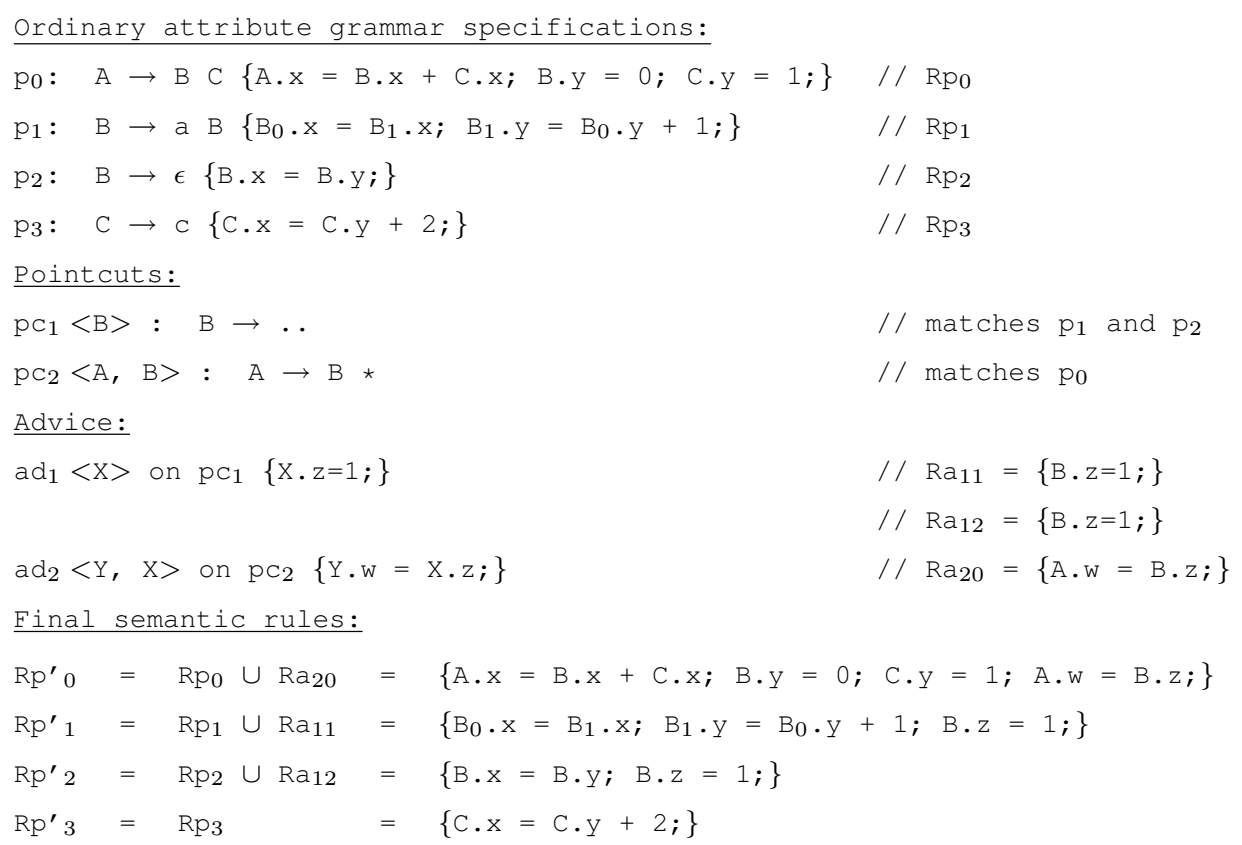

\subsection{AspectLISA constructs}

As seen from Fig. 1 and Fig. 2 LISA enables good modularity and extensibility of attribute grammar specifications. However, there are still situations when new semantic aspects crosscut basic modular structure. In other words, some semantic rules need to be repeated in different productions (e.g., semantic rule COMMAND.time $=1$; which has to be repeated several times in generalized production move of Robot Time language). To avoid this unpleasant situation, an aspect-oriented attribute grammar, as specified in subsection 4.2, has been incorporated into LISA language specifications. This extension is called AspectLISA. Join points in AspectLISA are static points in language specifications where additional semantic rules can be attached. These points can be syntactic production rules or generalised LISA rules. The production matching takes place on productions which are members of generalized LISA rules. One pointcut can match productions in different languages over the entire hierarchy of languages. For each pointcut we can define several advice which are parameterized semantic rules written as native Java assignment statements. In AOP, several different approaches of applying aspects to pointcuts exists, like before, after and around [11]. In AspectLISA there is only one way to apply advice on a specific pointcut, since attribute grammars are declarative and the order of equations in semantic rules is not important. Therefore, applying advice before/after a join point is not applicable.

The AspectLISA specification language, including apect-oriented features, pointcuts and advice, has the following parts (note how pointcuts and advice defined in section 4.2 are written in the LISA specification language): 


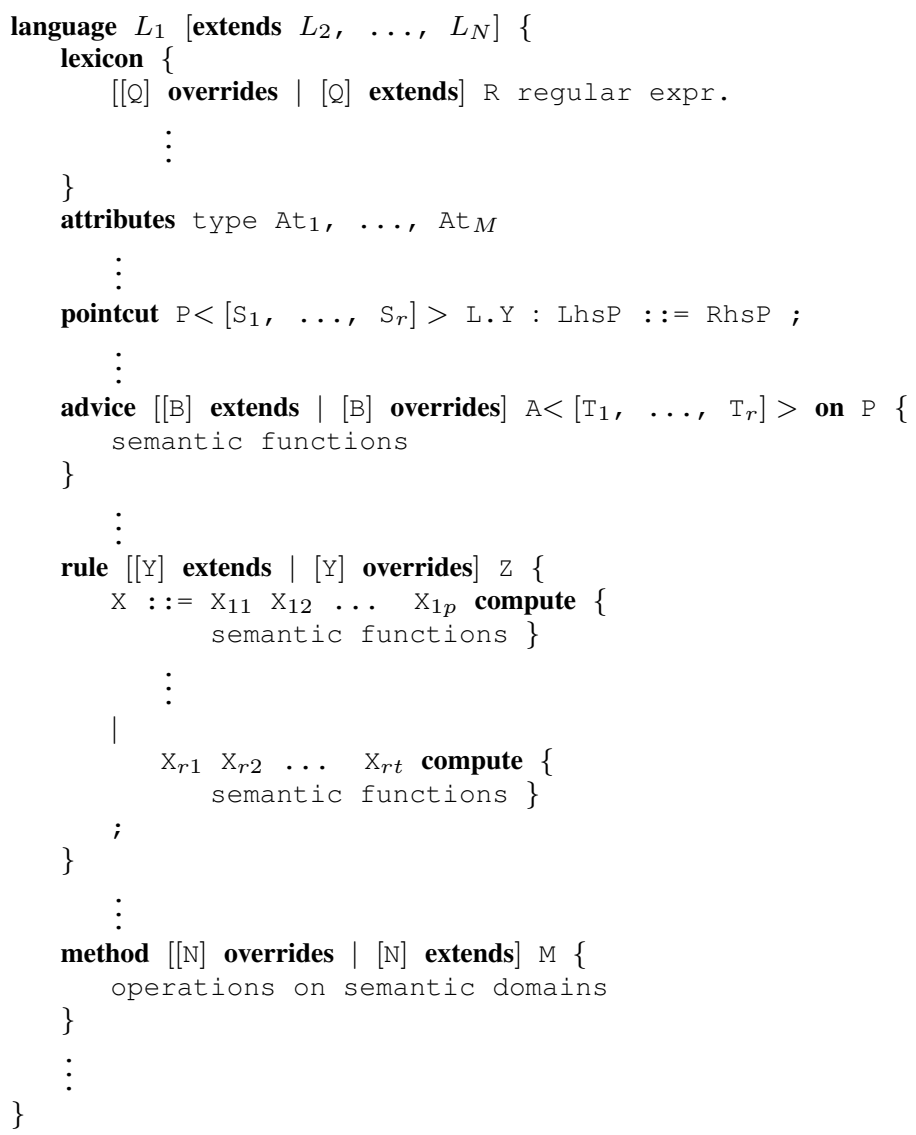

Symbols used in formal AspectLISA specifications above have following meaning:

- $L$ - language name,

- $Q$ and $R$ - regular expression name,

- At - attribute name,

- $P$ - pointcut name,

- $S$ - actual symbol,

- LhsP and RhsP - left and right-hand side of pointcut production,

- $A$-advice name,

- $T$ - formal symbol,

- $X$ - grammar symbol,

- $Y$ and $Z$ - grammar rules,

- $N$ and $M-$ method names.

This section focuses only on the new aspect-oriented features of the LISA specification language which are pointcuts and advice.

Pointcuts are defined using the reserved word pointcut. Each pointcut has a unique name and a list of actual parameters (terminals and non-terminals used 
in semantic functions of advice). As we already mentioned, join points are static points in language specifications where advice can be applied. In the pointcut definition one can use two wildcards. The wildcard '..' matches zero or more terminal or non-terminal symbols and can be used only to specify right-hand side matching rules. The wildcard ' $*$ ' is used to match parts or whole literal representing a symbol. To illustrate the AspectLISA pointcut model, we present some examples of pointcut specifications, defined over the Robot languages (Fig. 1, Fig. 2).

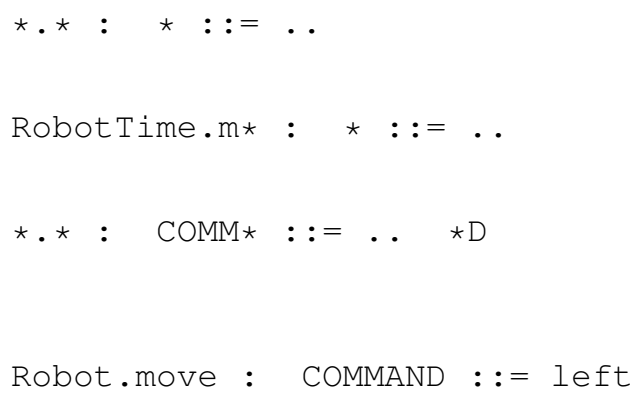

matches any production in any rule in all languages across current languge hieararchy

matches any production in all rules which start with $m$ in Robot Time language

matches all productions in any rule whose lefthand side symbol satisfy pattern "COMM*" and the right-hand side's last symbol ends with $D$

matches only a production COMMAND : := left in the rule move of Robot language

Advice in AspectLISA are additional semantics that can be appended at a specific join point. In order to increase reusability, advice are parameterized. Parameters can be terminal or non-terminal symbols and are evaluated at weaving time. Advice are defined using the reserved word advice and contain information about the pointcut where advice will appear. Below is an example of advice; more examples of advice and pointcuts are provided in section 5 .

pointcut SimpleCommand<COMMAND $>$ *move : COMMAND : := *;

advice SetTime $<\mathrm{C}>$ on SimpleCommand $\{$ C.time $=1$;

The result of weaving advice Set Time on pointcut SimpleCommand in the RobotTime language is an additional semantic rule COMMAND. $\mathrm{t} i \mathrm{me}=1$; in all productions of rule Robot.move. The notation is much simpler as in Fig. 2. The new aspect of the language, namely time calculation, is described at one place (advice) and is not repeated in several productions.

\subsection{AspectLISA inheritance}

The AspectLISA specification language is an extension of LISA with two new mechanisms (pointcuts and advice). Obviously, pointcuts and advice can also be inherited from ancestor specifications. Formal definition of multiple attribute grammar inheritance as described in [18] needs to be adopted. Due to lack of space in this paper only the formal definition of inheritance of pointcuts and advice are given. For theoretical background and further details readers are referred to [18].

Properties of aspect-oriented attribute grammars consist of lexical regular definitions, attribute definitions, rules which are generalized syntax rules that encapsulate semantic rules, pointcuts, advice and methods on semantic domains. 
Property $=$ RegdefName + AttributeName + RuleName + PointcutName+ AdviceName + MethodName

For each pointcut $p c$ in the language $l$, a Pointcuts $(l)(p c)$ is a finite set of matching productions $P$, that match to the pointcut $p c$, over the hierarchy of language $l$.

Pointcuts $:$ Language $\rightarrow$ PointcutName $\rightarrow$ MatchingProductionRules

Pointcuts $(l)(p c)=\left\{p_{i} \mid p_{i} \in P, p_{i}: X_{i 0} \rightarrow X_{i 1} X_{i 2} \ldots X_{i n}, \operatorname{match}\left(p_{i}, p c\right)\right\}$

For each advice $a d$ attached to pointcut $p c$ in the language $l$, Advice $(l)(a d)(p c)$ is a finite set (ProdSem) of pairs $\left(p, R_{p}\right)$, where $p$ is a production and $R_{p}$ is a union of finite set of semantic rules associated with the production $p$, and semantic rules (defined $R_{p}$ ) defined by advice $a d$, where formal symbols of advice are replaced by actual symbols defined in pointcut $p c$.

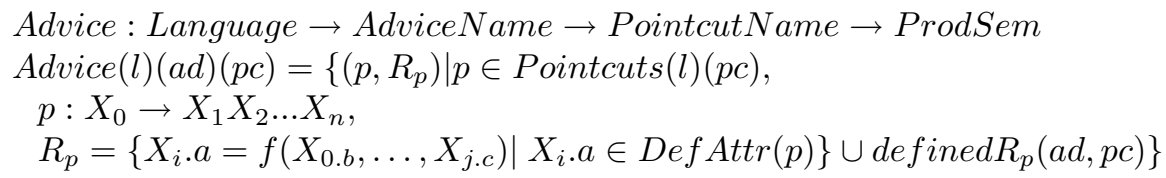

Multiple aspect-oriented attribute grammar inheritance is defined as follows.

Let Aspect $A G_{1}$, Aspect $A G_{2}, \ldots, A$ spect $A G_{m}$ be aspect-oriented attribute grammars formally defined as:

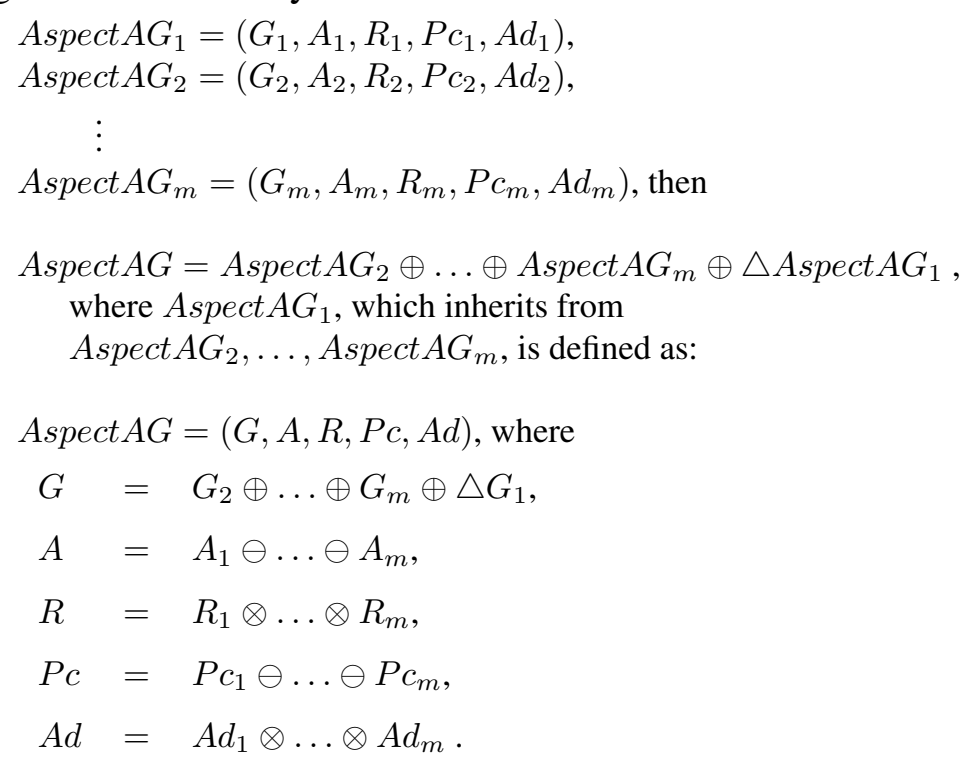

Therefore, inheritance on pointcuts is defined in a similar manner as for attributes [18]. Pointcuts as well as attributes cannot be extended, but can be inherited from ancestor attribute grammars. On the other hand, it is possible that some pointcut is redefined in current specifications which override pointcut specified in ancestor specifications. Inheritance on advice is defined in a similar manner as for semantic rules $R$ [18]. This should not be surprising, because advice are just additional semantic rules which need to be weaved at appropriate join points. 


\subsection{AspectLISA novelty}

AspectLISA is first specification language based on attribute grammars that use an explict pointcut model. Note that in JastAdd the pointcut model is implicit. The poincut model in AspectG is more complicated because syntax as well as semantic level are involved in the specification. This is due to using syntax directed translation instead of attribute grammars. AspectASF uses very simple pattern matching language where only labels and left-hand sides of equations written in ASF formalism can be matched. None of the existing systems enable inheritance on advice and pointcuts. Moreover, advice in AspectLISA are parameterized on grammar symbols and hence more reusable.

\section{Using AspectLISA}

Each LISA language specification is also a regular AspectLISA specification. In section 3.3 the RobotTime language has been specified as an extension of the Robot language using multiple attribute grammar inheritance. As can be noticed, semantic rule (COMMAND . $t$ ime $=1 ;$ ) has to be repeated in several productions (COMMAND $::=$ left, COMMAND $::=$ right, COMMAND $::=$ up, COMMAND $::=$ down). New semantics in the RobotTime language can be seen as a new aspect which crosscuts the language structure. Therefore, the RobotTime language can be better specified using aspect-oriented attribute grammars. The RobotTime language specifications written in AspectLISA are shown in Fig. 3. Note that four pointcuts have been specified which match all seven productions in the Robot language. For example, pointcut Begin matches production START : := begin COMMANDS end and pointcut $\mathrm{S}$ impleCommand matches productions COMMAND $::=$ left, COMMAND $::=$ right, COMMAND $::=$ up, and COMMAND $::=$ down. To each pointcut, advice is attached which define the new semantics of matched productions (e.g., semantic for simple command is that each command spent one time slot C.time $=1 ;$ ).

In [20], the RobotSpeed language has been defined as an extension of the RobotTime language. An additional speed construct has been added to the language such that the robot can now move with different speed. The RobotSpeed language can be specified purely with aspect-oriented techniques as shown in Fig. 4. Note that all pointcuts from the RobotTime language have been inherited. Only new advice have to be defined with additional semantics about speed of the movement. Hence, new advice extends previous advice that is inherited.

In Fig. 3 and Fig. 4 illustrative examples of AspectLISA are shown. The approach is scalable to larger languages and has been used in re-specifying the AspectCOOL language [3] which is an aspect-oriented extension of COOL (Class Object-Oriented Language $)^{5}$. Typical examples of aspects in language specifications can be additional code generation, different language extensions (e.g., excep-

${ }_{5}$ Our COOL language should not be confused with the early domain-specific aspect-oriented COOL language by Lopes. 


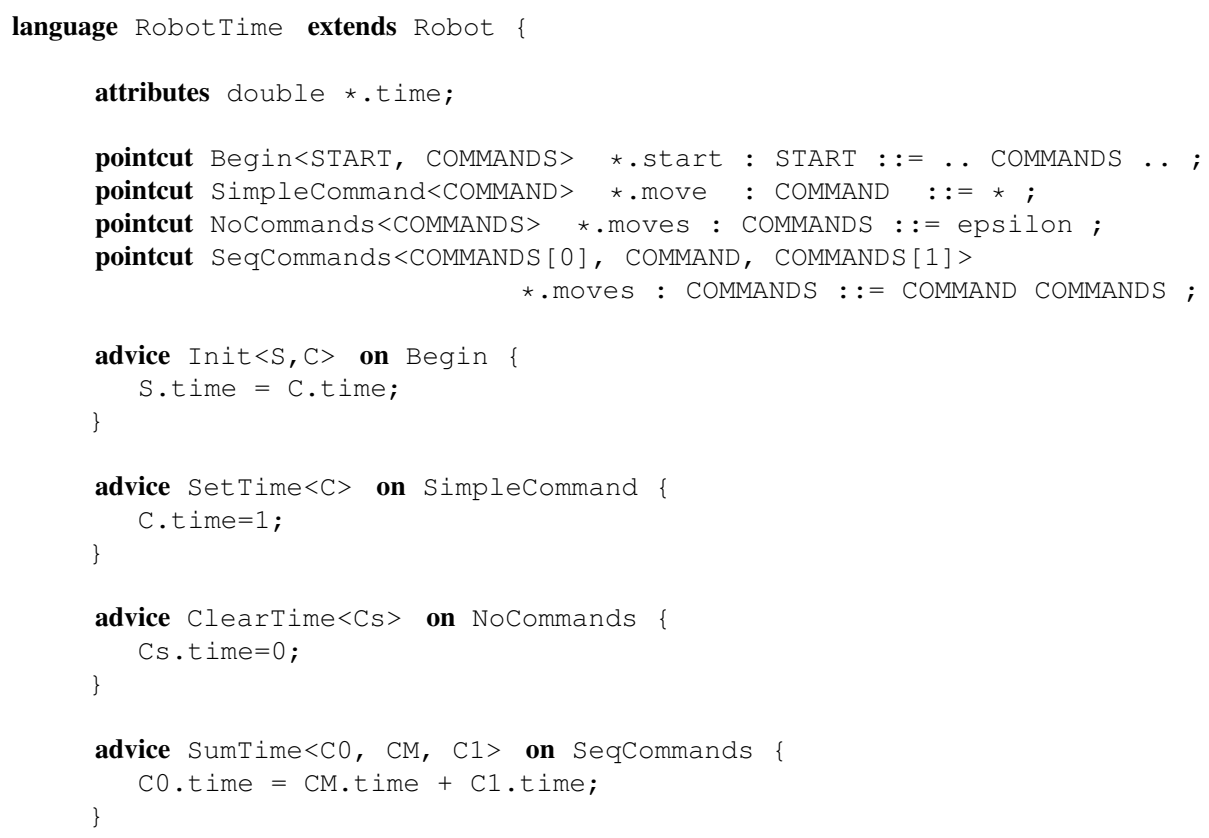

Fig. 3. RobotTime Language using AspectLISA

tion handling, aspects, new paradigms), language specification debugging, attribute tracking.

\section{Conclusion}

In the paper, aspect-oriented attribute grammars has been proposed and formally defined. The concept has been incorporated into AspectLISA, an aspect-oriented compiler generator based on attribute grammars. Aspect-oriented programming is a very promising approach and has been successfully used in tools for language definition and implementation. Some of the known contributions in this field were reviewed, as a motivation for our proposal. LISA already has mechanisms to support inheritance and modularity. These mechanisms support nicely the notion of object-oriented aspects; on the other side, adding aspects will allow to write simpler specifications avoiding, for example, the repetition of semantic rules. The challenge in programming language definition is also to support reusability and extensibility: aspects will reinforce these features. Aspect-oriented features of the AspectLISA tool increase modularity since different concepts of programming language can be designed and implemented separately in different modules. These modules are also more reusable due to inheritance, which is successfully incorporated into our tool.

\section{Acknowledgements}

We would like to thank Jeff Gray for useful comments on earlier versions and Mitja Lenič for ideas and tips on implementation. 


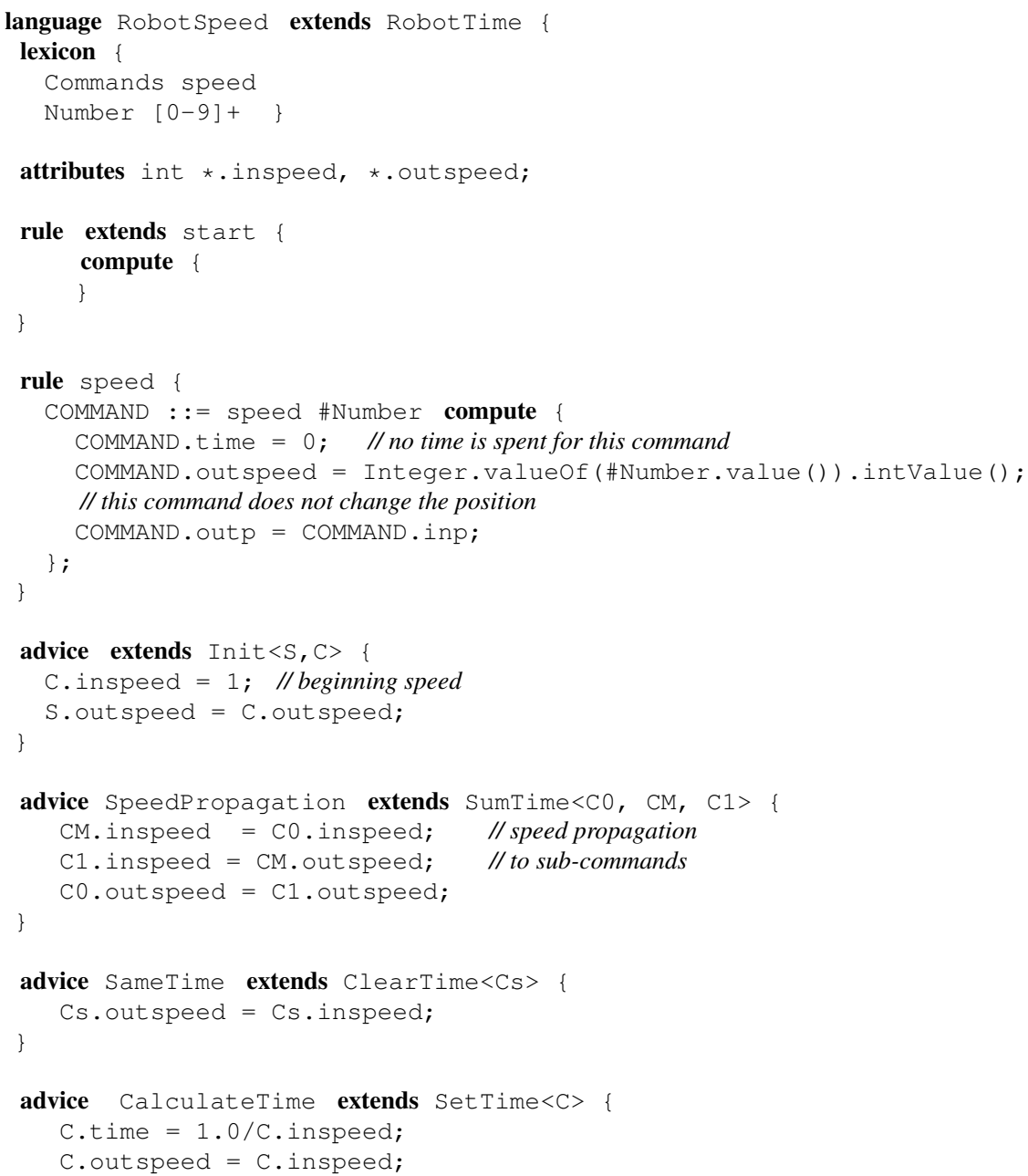

Fig. 4. RobotSpeed Language using AspectLISA

\section{References}

[1] ANTLR - ANother Tool for Language Recognition. http://www.antlr.org, 2006.

[2] AspectG. http://www.cis.uab.edu/wuh/ddf/index.html, 2006.

[3] E. Avdičaušević, M. Lenič M. Mernik, and V. Žumer. AspectCOOL: An experiment in design and implementation of aspect-oriented language. ACM SIGPLAN Notices, 36(12):84-94, December 2001.

[4] O. de Moor, S. L. Peyton Jones, and E. Van Wyk. Aspect-oriented compilers. In Generative and Component-Based Software Engineering (GCSE), pages 121-133, 1999.

[5] E. W. Dijkstra. A Discipline of Programming. Prentice-Hall, 1976.

[6] R.W. Gray, V.P. Heuring, S.P. Levi, A.M. Sloane, and W.M. Waite. Eli: A complete, flexible compiler construction system. Communications of the ACM, 35(2):121-131, 1992. 
[7] G. Hedin and E. Magnusson. JastAdd: an aspect-oriented compiler construction system. Science of Computer Programming, 47(1):37-58, 2003.

[8] P. Henriques, M. Varanda Pereira, M. Mernik, M. Lenič, J. Gray, and H. Wu. Automatic generation of language-based tools using LISA. IEE Proceedings Software Engineering, 152(2):54-69, April 2005.

[9] M. Jourdan, D. Parigot, C. Julie, O. Durin, and C. Le Bellec. Design, implementation and evaluation of FNC-2 attribute grammar system. In Proc. of the ACM Sigplan'90 Conference on Programming Language Design and Implementation, pages 209-222, 1990.

[10] K. Kalleberg and E. Visser. Combining aspect-oriented and strategic programming. In N.M.-O. Horatiu Cirstea, editor, Proceedings of the 6th International Workshop of Rule-Based Programming (RULE). ENTCS, Nara, Japan, Elsevier, April 2005.

[11] G. Kiczales, E. Hilsdale, J. Hugunin, M. Kersten, J. Palm, and W. G. Griswold. Getting started with AspectJ. Communications of the ACM (Special issue on Aspect-Oriented Programming), 44(10):59-65, October 2001.

[12] G. Kiczales, J. Lamping, A. Mendhekar, C. Maeda, C. Lopes, J.-M. Loingtier, and J. Irwin. Aspect-oriented programming. In M. Aksit and S. Matsuoka, editors, ECOOP97 Object-Oriented Programming, Lecture Notes in Computer Science, volume 1241, pages 220-242. Springer-Verlag, 1997.

[13] P. Klint, T.van der Storm, and J.J. Vinju. Term rewriting meets aspect-oriented programming. Technical report, Centrum voor Wiskunde en Informatica, 2004.

[14] D. E. Knuth. Semantics of context-free languages. Mathematical Systems Theory, 2(2):127-145, 1968.

[15] J. Kollár and M. Tóth. Temporal logic for pointcut definitions in AOP. Acta Electrotechnica et Informatica, 5(2):15 - 22, 2005.

[16] D. Lohmann, G. Blaschke, and O. Spinczyk. Generic advice: On the combination of AOP with generative programming in aspectC++. In GPCE, pages 55-74, 2004.

[17] M. Mernik, J. Heering, and A. Sloane. When and how to develop domain-specific languages. ACM Computing Surveys, 37(4), 2005. To appear.

[18] M. Mernik, M. Lenič, E. Avdičaušević, and V. Žumer. Multiple Attribute Grammar Inheritance. Informatica, 24(3):319-328, September 2000.

[19] M. Mernik, X. Wu, and B. Bryant. Object-oriented language specifications: Current status and future trends. In ECOOP Workshop: Evolution and Reuse of Language Specifications for DSLs (ERLS), 2004.

[20] M. Mernik and V. Žumer. Incremental programming language development. Computer Languages, Systems and Structures, (31):1-16, 2005.

[21] J. Paakki. Attribute Grammar Paradigms - A High-Level Methodology in Language Implementation. ACM Computing Surveys, 27(2):196 - 255, 1995. 
[22] D. L. Parnas. On the criteria to be used in decomposing systems into modules. Communications of the ACM, 15(12):1053-1058, December 1972.

[23] K. Slonneger and B. L. Kurtz. Formal Syntax and Semantics of Programming Languages. Addison-Wesley, 1995.

[24] C. A. Szyperski. Component Software - Beyond Object-Oriented Programming. Addison Wesley, Second edition, 2002.

[25] M. G. J. van den Brand, A. van Deursen, J. Heering, H. A. de Jong, M. de Jonge, T. Kuipers, P. Klint, L. Moonen, P. A. Olivier, J. Scheerder, J. J. Vinju, E. Visser, and J. Visser. The ASF + SDF meta-environment: A component-based language development environment. Lecture Notes in Computer Science, 2027:365-370, 2001.

[26] H. Wu, J. Gray, S. Roychoudhury, and M. Mernik. Weaving a debugging aspect into domain-specific language grammars. In SAC '05: Proceedings of the 2005 ACM symposium on Applied computing, pages 1370-1374, New York, NY, USA, 2005. ACM Press.

[27] E. Van Wyk. Aspects as modular language extensions. Electronic Notes in Theoretical Computer Science, 82(3), 2003. 